\title{
Glutest Neo Super-a New Handheld Blood Glucose Meter-Corrects for the Effects of the Hematocrit Values in Both Hematocrit-Adjusted Samples and Samples Obtained from Anemic Patients
}

\author{
Fumiatsu Yakushiji, M.D., Ph.D., ${ }^{1}$ Hiroshi Fujita, M.D., Ph.D., ${ }^{2}$ Hiroshi Suzuki, M.Tech., ${ }^{3}$ \\ Ritsuko Joukyu, M.Tech., ${ }^{3}$ Mutsuko Yasuda, M.D., ${ }^{1}$ Yoshiyasu Terayama, M.Sc., ${ }^{4}$ \\ Kaoru Nagasawa, M.D., Ph.D., ${ }^{5}$ Akira Ohwada, M.D., Ph.D., ${ }^{1}$ Ken Taniguchi, M.D., Ph.D., D.D.S., ${ }^{1}$ \\ Kazuhiko Fujiki, M.D., Ph.D., ${ }^{1}$ Masako Shimojo, M.D., Ph.D., ${ }^{6}$ and Hiroyuki Kinoshita, M.D., Ph.D. ${ }^{1}$
}

\begin{abstract}
Background: Handheld blood glucose (BG) meters are convenient tools that are widely used to measure the BG levels. However, the hematocrit (Hct) value has been identified as a confounding factor for accurate BG measurement. Some BG meters are equipped with an Hct-correcting feature, whose effectiveness has been tested previously. Nevertheless, the measurements yielded by many BG meters are confounded by the Hct values. Recently, a new BG meter equipped with an Hct-correcting feature has been developed; however, its effectiveness has not yet been confirmed.

Study Design: Venous blood samples were collected from two healthy volunteers, and the Hct values in the samples were adjusted to approximately $0 \%, 10 \%, 20 \%, 30 \%, 40 \%, 50 \%$, and $60 \%$. Further, venous blood samples were collected from 10 anemic patients (Hct $<40 \%$ ). The whole BG (WBG) levels in the samples were measured using two devices-the new BG meter (Glutest Neo ${ }^{\circledR}$ Super [Sanwa Kagaku Kenkyusho Co. Ltd., Nagoya, Japan]) and a standard BG meter (OneTouch ${ }^{\circledR}$ Ultra ${ }^{\circledR}$ [Life Scan Inc., Milpitas, CA]). For reference, plasma glucose (PG) levels were measured using a machine at our hospital laboratory (GA08 [A\&T Co., Kanagawa, Japan]). The bias in the measurements was calculated as follows: bias $=([W B G-P G] / P G) \times 100$. Further, the correlation between the Hct values and the bias was assessed by performing linear regression analysis.

Results: In both the Hct-adjusted samples and the samples obtained from anemic patients, the WBG levels measured using Glutest Neo Super were minimally affected by the Hct values, while those measured using OneTouch Ultra were affected by the Hct values to a statistically significant extent.

Conclusions: The Hct-correcting feature of the new BG meter Glutest Neo Super was effective. The use of this new device for BG measurements may lead to more appropriate treatment selection.
\end{abstract}

\section{Introduction}

$\mathbf{H}$ ANDHELD BLOOD GLUCOSE (BG) meters are convenient tools that are widely used for measuring the BG levels of diabetes patients. Since BG meters are more efficient than laboratory machines in calculating the glucose value, they are often employed not only in homes but also in hospitals. However, since the 1960s, the hematocrit (Hct) value has been recognized as a confounding factor for accurate BG measurements. ${ }^{1}$ Depending on their product description, some BG meters are equipped with an Hct-correcting feature, whose efficiency has been tested in some previous studies. ${ }^{2,3}$ Nevertheless, the results yielded by many types of BG meters developed thus far are confounded by the Hct values. ${ }^{4,5} \mathrm{Nu}$ merical formulae for Hct correction have been proposed in some previous articles. ${ }^{6,7}$

Departments of ${ }^{1}$ Internal Medicine and ${ }^{2}$ Transfusion Medicine and ${ }^{3}$ Clinical Laboratory and ${ }^{4}$ Pharmacy Departments, Tokyo Metropolitan Bokutoh Hospital; and ${ }^{5}$ Department of Endocrinology and Metabolism, Toranomon Hospital, Tokyo, Japan.

${ }^{6}$ Internal Medicine, Kanagawa Prefectual Shiomidai Hospital, Yokohama, Japan. 
In 2007, a new type of BG meter equipped with an Hctcorrecting feature was developed in Japan; however, its effectiveness has not yet been confirmed.

In this study, we prepared blood samples with adjusted Hct values (approximately $0 \%, 10 \%, 20 \%, 30 \%, 40 \%, 50 \%$, and $60 \%$ ) and obtained blood samples from anemic patients (Hct $<40 \%$ ). Further, we compared the new BG meter with a standard BG meter. For reference, plasma glucose (PG) values were measured using a machine at our hospital laboratory. The results revealed that the new BG meter had excellent efficiency with regard to Hct correction.

\section{Subjects and Methods}

\section{Samples}

Hct-adjusted blood samples. Venous blood samples were collected from two healthy volunteers and were heparinized and centrifuged to obtain blood cells and plasma. These fractions were used to prepare samples with Hct values adjusted to approximately $0 \%, 10 \%, 20 \%, 30 \%, 40 \%, 50 \%$, and $60 \%$. The samples were then used to measure the whole BG (WBG) levels. The Hct value for each sample was precisely measured by using an Hct tube. The samples were centrifuged to obtain plasma, which was subsequently used to measure the PG levels.

Blood samples from anemic patients. Written informed consent was obtained from 10 anemic patients (Hct $<40 \%$ ), after which their venous blood samples were collected and heparinized. These samples were used to measure the WBG levels. The Hct value for each sample was measured using an Hct tube and was found to be low $(<40 \%)$ in all the samples. The samples were centrifuged to obtain plasma, which was subsequently used for PG measurements.

\section{$B G$ meters}

Glutest Neo ${ }^{\circledR}$ Super (Sanwa Kagaku Kenkyusho Co. Ltd., Nagoya, Japan), developed in 2007, is a new type of BG meter with a glucose dehydrogenase-flavin adenine dinucleotide electrode. The device is equipped with a program functioning for Hct correction. Thus, this BG meter can correct glucose values in response variations in the Hct values.

OneTouch $^{\circledR}$ Ultra $^{\circledR}$ (Life Scan Inc., Milpitas, CA) is a standard BG meter with a glucose oxidase-type electrode. According to its instruction manual, this device functions with adequate efficiency when the Hct value is in the range of 30$55 \%$.

The automatic sugar analyzer (GA08 [A\&T Co., Kanagawa, Japan]), presently available at our hospital laboratory, was used to determine PG levels for reference.

\section{Measurements and data analysis}

Comparison of WBG levels in Hct-adjusted blood samples. The WBG levels in the Hct-adjusted blood samples were measured using the two BG meters; the measurements were performed in triplicate. For reference, PG levels were measured using the GA08.

The bias in the measurements was calculated by using the following equation: Bias $=([$ observed $W B G-P G] / P G) \times 100$.

The correlation between the Hct values and the bias was assessed by performing linear regression analysis.
Comparison of WBG levels in blood samples obtained from anemic patients. The two BG meters were used to measure the WBG levels in blood samples obtained from the anemic patients. For reference, PG levels were measured using the GA08.

The bias in the measurements and the correlation between the Hct levels and the bias were calculated using the method described for Hct-adjusted samples. All statistical procedures were conducted using Statview version 5.0 software (SAS Institute Inc., Cary, NC), and significance was defined as $P<0.05$.

\section{Results}

\section{Comparison of WBG levels in Hct-adjusted blood samples}

Figure 1 shows the results of the comparison of the WBG levels measured in the Hct-adjusted blood samples by using the two BG meters. The linear equation, correlation coefficient, and results of $t$ tests performed to determine the slope of the linear equation were as follows:

$$
\begin{aligned}
& y=0.092 x-0.011, R=0.3104, P=0.28 \\
& \text { for Glutest Neo Super } \\
& y=-1.462 x+0.456, R=-0.9271, P<0.001 \\
& \text { for OneTouch Ultra }
\end{aligned}
$$

These results indicated that the WBG levels measured in the Hct-adjusted samples by using Glutest Neo Super were minimally affected by the Hct values, while those measured using OneTouch Ultra were affected by the Hct values to a statistically significant extent. The WBG levels were overestimated when the Hct values were low but were underestimated when the Hct values were high.

\section{Comparison of WBG levels in blood samples of anemic patients}

The characteristics of the 10 anemic patients (nine men and one woman) were as follows: mean age \pm standard deviation

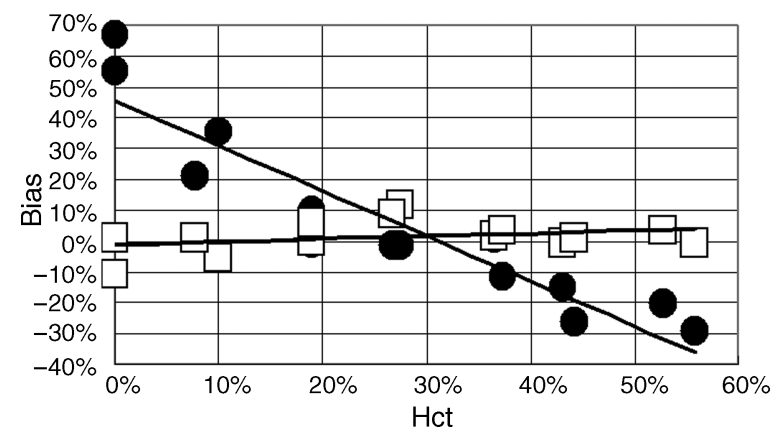

FIG. 1. Correlation between the WBG-PG bias and the Hct values determined for Hct-adjusted samples. Bias is plotted against Hct values by the equation Bias $=([W B G-P G] /$ $P G) \times 100$. The $B G$ meter used, the linear regression equation, the correlation coefficient $(R)$, and the results of the $t$ test $(P$ value) for the slope of the linear equation are as follows: Glutest Neo Super $(\square), y=0.092 x-0.011, R=0.3104, P=0.28$; OneTouch Ultra $(\bullet), y=-1.462 x+0.456, \quad R=-0.9271$, $P<0.001$. 
(SD), 53.1 \pm 5.3 years (range, 29-78 years); mean Hct value, $28.2 \pm 1.7 \%$ (range, 20.6-39.4\%); and mean venous PG value, $6.48 \pm 0.66 \mathrm{mmol} / \mathrm{L}(116.7 \pm 11.9 \mathrm{mg} / \mathrm{dL})$ (range, $4.61-11.32$ $\mathrm{mmol} / \mathrm{L}$ [83-204 mg/dL]). The patient population comprised eight patients with hematological disease, one patient with gastric cancer, and one patient with duodenal ulcers. Of the 10 patients, two were diagnosed with diabetes mellitus.

Figure 2 shows the results of the comparison of the WBG levels in the blood samples of the anemic patients, as determined using the two BG meters. The linear equation, correlation coefficient, and results of $t$ tests performed to determine the slope of the linear equation were as follows:

$$
\begin{aligned}
& y=-0.014 x-0.090, R=-0.022, P=0.9510 \\
& \text { for Glutest Neo Super } \\
& y=-1.947 x+0.608, R=-0.7288, P=0.0168 \\
& \text { for OneTouch Ultra }
\end{aligned}
$$

These results indicated that the WBG levels measured in the blood samples of the anemic patients by using Glutest Neo Super were minimally affected by the Hct values, while those measured using OneTouch Ultra were affected by the Hct values to a statistically significant extent. The WBG values were overestimated when the Hct values were low but were underestimated when the Hct values were high. These results were consistent with those obtained for the Hct-adjusted samples, as described above.

On the basis of the results described in this study, i.e., those obtained for the Hct-adjusted samples and the blood samples from anemic patients, we concluded that the Hct-correcting feature of Glutest Neo Super is effective.

\section{Conclusions}

Here, we demonstrated that the WBG levels measured in both Hct-adjusted samples and samples from anemic patients by using the new BG meter-Glutest Neo Super-are mini-

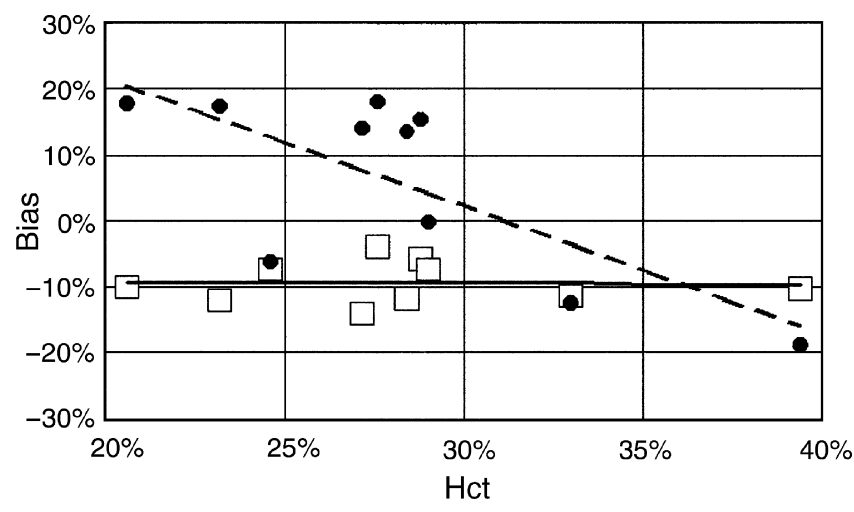

FIG. 2. Correlation between the WBG-PG bias and the Hct values determined for samples obtained from anemic patients. Bias is plotted against Hct values by the equation Bias $=([W B G-P G] / P G) \times 100$. The BG meter used, the linear regression equation, the correlation coefficient $(R)$, and the results of the $t$ test ( $P$ value) for the slope of the linear equation are as follows: Glutest Neo Super $(\square)$, $y=-0.014 x-0.090, R=-0.022, P=0.9510$; OneTouch Ultra (•), $y=-1.947 x+0.608, R=-0.7288, P=0.0168$. mally affected by the Hct values, while those measured using the standard BG meter-OneTouch Ultra-are affected by the Hct values to a statistically significant extent.

In the present study, the standard BG meter overestimated the WBG level in cases where the Hct value was low. A similar tendency has been described in many previous reports of studies performed using other conventional BG meters. $5,8,9$ According to our results, there was a positive bias of approximately $30 \%$ in the measurements obtained when the Hct value was $10 \%$. This indicates that a PG level of $2.78 \mathrm{mmol} / \mathrm{L}$ $(50 \mathrm{mg} / \mathrm{dL})$ corresponds to a WBG level of $3.61 \mathrm{mmol} / \mathrm{L}$ $(65 \mathrm{mg} / \mathrm{dL})$ when the Hct value is $10 \%$. Since patients with low Hct values are prone to developing infections, it is important to control the BG levels in order to prevent the development and progression of infection. Moreover, in patients with severe bleeding or those undergoing surgery, by much intravenous drip, the Hct value is considerably reduced. If a standard BG meter is used in the case of these patients, hypoglycemia may be missed, and this may have persistent adverse effects.

In addition, when the standard BG meter was used in the case of high Hct values, the WBG levels were underestimated. A similar tendency has been described in the reports of many previous studies performed using other conventional BG meters. ${ }^{5,9,10}$ According to our results, there was a negative bias of approximately $30 \%$ in the measurements obtained when the Hct value was $50 \%$. This indicates that a PG level of $27.8 \mathrm{mmol} / \mathrm{L}(500 \mathrm{mg} / \mathrm{dL})$ corresponds to a WBG level of $19.4 \mathrm{mmol} / \mathrm{L}(350 \mathrm{mg} / \mathrm{dL})$ when the Hct value is $50 \%$. In general, the Hct values are elevated in patients with diabetic ketoacidosis or hyperosmolar hyperglycemic state because of severe dehydration. If a standard BG meter is used for such diabetes patients with emergency conditions, the WBG may be underestimated, and, consequently, the dose of insulin administered may be not be sufficient to bring about an improvement in the patients' condition.

PG measurements obtained using machines in hospital laboratories are the most reliable; however, the process involved is time consuming. On the other hand, handheld BG meters are useful for rapid measurement of the WBG levels. In the present study, Glutest Neo Super yielded excellent results with regard to Hct correction. Therefore, in cases where frequent BG monitoring is required, especially those involving patients with abnormal Hct values, the use of the new BG meter instead of conventional BG meters may lead to more appropriate treatment selection.

The limitations of the present study are as follows. First, we measured the venous WBG levels using BG meters, which are designed mainly for measuring these levels in samples obtained from arterial capillaries of the finger. Moreover, consistent with a previous report, the venous WBG levels were found to be approximately $10-15 \%$ lower than the PG levels. ${ }^{11}$ Nevertheless, many previous studies performed to verify the efficiency of BG meters have applied strategies identical to those used in the present study. Second, we concluded that the new BG meter is effective, solely on the basis of comparison with one other BG meter. However, as reported previously, many studies performed using conventional BG meters and methods identical to those used in the present study have reported that the Hct values have similar effects on the measurements. ${ }^{5,8-10}$ Some of the results obtained for BG meters tested in previous studies are as follows: $y=-1.161 x+0.339$, 
$R=-0.693, P<0.001$ for Glutest Neo (Sanwa Kagaku Kenkyusho Co. Ltd.) and $y=-1.661 x+0.516, \quad R=-0.872$, $P<0.001$ for Precision ${ }^{\circledR}$ Xceed $^{\circledR}$ (Abbott Diabetes Care, Abbott Park, IL). ${ }^{5}$ Similar to the results of our present study, these results also indicated that the WBG levels are overestimated when the Hct value is low and underestimated when the Hct value is high. Therefore, it can be considered that the new $\mathrm{BG}$ meter can provide more reliable glucose measurements than conventional BG meters.

According to the information provided by Sanwa Kagaku Kenkyusho Co. Ltd., the principle on which the Hctcorrecting feature of Glutest Neo Super functions is as follows. The device contains a sensor equipped with an electrode for measuring the Hct value. Blood cells introduced into this device are exposed to redox conditions. Further, the Hctdependent redox potential is measured under a maximum voltage of $6 \mathrm{~V}$, and the glucose concentration is corrected in the presence of this current. Additional information on this principle is available in the patent documents. ${ }^{12,13}$

Thus far, many factors such as uric acid and bilirubin levels, body temperature, humidity, and the aspirin and vitamin C levels have been reported to affect the measurements yielded by BG meters. ${ }^{14}$ Further research is required for the development of quick and reliable techniques for measuring BG.

\section{Acknowledgments}

This study was supported by a Grant-in-Aid from the Tokyo Metropolitan Government Clinical Research Foundation.

\section{Author Disclosure Statement}

No competing financial interests exist.

\section{References}

1. Dilon RS, Mawr B: Importance of the hematocrit in interpretation of blood sugar. Diabetes 1965;14:672-674.

2. Makoto T, Isao K, Katsuhiro K, Izumi T, Tadao H, Gen Y, Takashi K, Yoshiaki K, Hideshi K, Masamichi K, Tomohiko T, Eiichi M, Junko O, Zenji M, Motoaki S: [Report of the Committee on Standardization of Laboratory Testing Related to Diabetes Mellitus: usefulness of venous blood method as a standardized comparative method for blood glucose measurement using devices]. J Jpn Diabetes Soc. 2002;45:825-834.

3. Rao LV, Jakubiak F, Sidwell JS, Winkelman JW, Snyder ML: Accuracy evaluation of the new glucometer with automated hematocrit measurement and correction. Clin Chim Acta 2005;356:178-183.
4. Karon BS, Griesmann L, Scott R, Bryant SC, Dubois JA, Shirey TL, Presti S, Santrach PJ: Evaluation of the impact of hematocrit and other interference on the accuracy of hospital-based glucose meters. Diabetes Technol Ther 2008;10:111-120.

5. Yakushiji F, Yasuda M, Taniguchi K, Fujiki K, Oowada A, Akiyama N, Suzuki H, Jokyu R, Terayama Y, Shimojo M: [Risk of blood glucose measurements with handheld meters in anemia patient]. J Jpn Diabetes Soc 2008;51:837-843.

6. Man EA, Salinas J, Pidcoke HF, Wolf SE, Holcomb JB, Wade $\mathrm{CE}$ : Error rates resulting from anemia can be corrected in multiple commonly used point-of-care glucometers. J Trauma 2008;64:15-21.

7. Maser RE, Butler MA, DeCherney GS: Use of arterial blood with bedside glucose reflectance meters in an intensive care unit: are they accurate? Crit Care Med 1994;22:595599.

8. Balion C, Grey V, Ismaila A, Blatz S, Seidlitz W: Screening for hypoglycemia at the bedside in the neonatal intensive care unit (NICU) with the Abbott PCx glucose meter. BMC Pediatr 2006;6:28.

9. Tang Z, Lee JH, Louie RF, Kost GJ: Effects of different hematocrit levels on glucose measurements with handheld meters for point-of-care testing. Arch Pathol Lab Med 2000;124:1135-1140.

10. Kaplan M, Blondheim O, Alon I, Eylath U, Trestian S, Eidelman AI: Screening for hypoglycemia with plasma in neonatal blood of high hematocrit value. Crit Care Med 1989;17:279-282.

11. Hussain K, Sharief N: The inaccuracy of venous and capillary blood glucose measurement using reagent strips in the newborn period and the effect of haematocrit. Early Hum Dev 2000;57:111-121.

12. Fujiwara M, Sinnno T, Ikeda S: Blood component measuring method, sensor used therefor, and measuring instrument. Japanese Patent P2005-515983. June 16, 2005.

13. Fujiwara M, Sinnno T, Ikeda S: Blood component measuring method, sensor used therefor, and measuring instrument. International Patent WO2005/054840. June 16, 2005.

14. Hönes J, Müller P, Surridge N: The technology behind glucose meters: test strips. Diabetes Technol Ther 2008; 10(Suppl 1):S-10-S-26.

Address reprint requests to: Fumiatsu Yakushiji, M.D., Ph.D. Department of Internal Medicine Tokyo Metropolitan Bokutoh Hospital 4-23-15 Koutohbashi, Sumida-ku Tokyo 130-8575, Japan

E-mail: clinic@nifty.com 